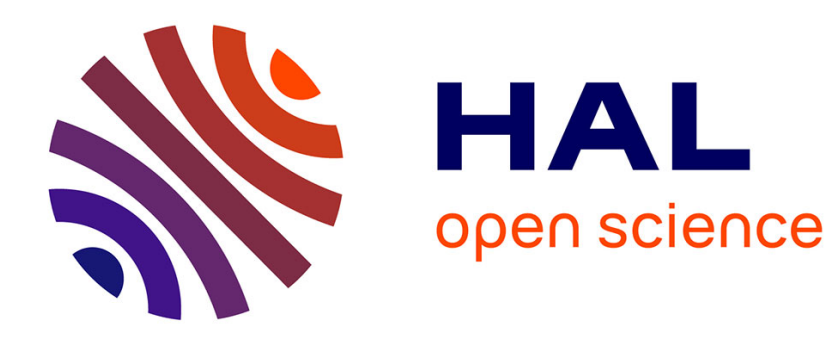

\title{
Des multiculturalismes en Europe au multiculturalisme européen
}

Riva Kastoryano

\section{To cite this version:}

Riva Kastoryano. Des multiculturalismes en Europe au multiculturalisme européen. Politique étrangère, 2000, 65 (1), pp.163-178. 10.3406/polit.2000.4919 . hal-01011199

\section{HAL Id: hal-01011199 \\ https://hal-sciencespo.archives-ouvertes.fr/hal-01011199}

Submitted on 23 Jun 2014

HAL is a multi-disciplinary open access archive for the deposit and dissemination of scientific research documents, whether they are published or not. The documents may come from teaching and research institutions in France or abroad, or from public or private research centers.
L'archive ouverte pluridisciplinaire HAL, est destinée au dépôt et à la diffusion de documents scientifiques de niveau recherche, publiés ou non, émanant des établissements d'enseignement et de recherche français ou étrangers, des laboratoires publics ou privés. 


\section{Des multiculturalismes en Europe au multiculturalisme européen} In: Politique étrangère $\mathrm{N}^{\circ} 1$ - 2000 - 65e année pp. 163-178.

Citer ce document / Cite this document :

Kastoryano. Des multiculturalismes en Europe au multiculturalisme européen. In: Politique étrangère $N^{\circ} 1-2000-65 e$ année pp. 163-178.

doi : $10.3406 /$ polit.2000.4919

http://www.persee.fr/web/revues/home/prescript/article/polit_0032-342X_2000_num_65_1_4919 


\section{Résumé}

Riva Kastoryano livre ici une réflexion sur le multiculturalisme plus précisément en Europe, dans les différents pays, mais aussi au niveau de l'Union européenne. En effet, le multiculturalisme devient un thème de plus en plus important dans des sociétés industrielles plurielles qui doivent faire face à une diversité culturelle. II fait également l'objet d'un vif débat entre les tenants du respect de la différence, à travers la mise en place de "politiques de la reconnaissance », et ceux défendant l'unité du groupe considéré. Bien que l'expérience des pays européens apparaisse diverse de ce point de vue en fonction de leurs traditions politiques et culturelles, et de leurs particularités régionales et linguistiques, l'auteur considère qu'ils tendent à se rapprocher en matière de " multiculturalisme appliqué ». Elle pense que cette gestion de la diversité doit être étendue à l'échelle de I'Union, compte tenu de son pluralisme culturel intrinsèque, et pourrait constituer le socle de l'identité européenne.

\section{Abstract}

From Forms of Multiculturalism in Europe to European Multiculturalism, by Riva Kastoryano

Whether it is accepted, challenged or rejected, multiculturalism has imposed itself as an historic concept of changing both the institutions and the political standards in the Western democracies. It may be regarded as a - pragmatic or ideological — response to the management of diversity that is specifie to any industrial society and to the ways and means of integrating it in the political community. Its application is at the heart of the interactions between immigrant or minority populations and States, leading the latter to conduct "identity negotiations » on either side. Seen from this perspective, multiculturalism could constitute a theory that allows Nation-States to strike a new balance between emerging community structures and national institutions. The same question arises in the context of the building of Europe: multiculturalism, a fact due originally to the diverse identities of the Nation-States of which Europe is made up, could be analyzed as a founding theory of a European identity. 


\section{Riva KASTORYANO}

\section{Des multiculturalismes en Europe au multiculturalisme européen}

Riva Kastoryano livre ici une réflexion sur le multiculturalisme plus précisément en Europe, dans les différents pays, mais aussi au niveau de l'Union européenne. En effet, le multiculturalisme devient un thème de plus en plus important dans des sociétés industrielles plurielles qui doivent faire face à une diversité culturelle. Il fait également l'objet d'un vif débat entre les tenants du respect de la différence, à travers la mise en place de "politiques de la reconnaissance", et ceux défendant l'unité du groupe considéré. Bien que l'expérience des pays européens apparaisse diverse de ce point de vue en fonction de leurs traditions politiques et culturelles, et de leurs particularités régionales et linguistiques, l'auteur considère quils tendent à se rapprocher en matière de "multiculturalisme appliqué". Elle pense que cette gestion de la diversité doit être étendue à l'échelle de l'Union, compte tenu de son pluralisme culturel intrinsèque, et pourrait constituer le socle de l'identité européenne.

Politique étrangère

$\mathrm{D}$ epuis que le mot multiculturalisme s'est installé dans les débats publics, il se prête à toutes sortes d'usages, rappelle Michael Walzer ${ }^{1}$. En effet, rares sont les mots en sciences sociales qui ont connu un usage aussi diversifié. Non seulement le concept de multiculturalisme a voyagé à travers les démocraties occidentales, voire au-delà ; mais aussi, à l'intérieur d'un même contexte national, il est source de controverses quant à sa définition et à son interprétation.

De toute évidence, le terme prête à confusion. Il peut être défini comme une situation relevant d'une diversité culturelle, d'un pluralisme propre à toute société industrielle. Ce constat de fait laisse sa

Riva Kastoryano est chargée de recherche au CNRS, Centre d'études et de recherches internationales (CERI, FNSP).

1. "Le multiculturalisme au cœeur", entretien avec Michael Waizer réalisé par Riva Kastoryano, Laurent Bouvet et Christophe Jaffrelot. Critique internationale, n 3, printemps 1999, p. 55-63. 
place à une idéologie lorsque la diversité donne lieu à des expressions identitaires particularistes débordant dans la sphère publique, remettant ainsi en cause l'unité culturelle et l'intégrité des nations. Dans tous les cas, le multiculturalisme, accepté, contesté ou rejeté, s'est imposé comme un concept historique de changement à la fois des institutions et des normes politiques dans les démocraties libérales occidentales.

Le multiculturalisme se présente comme un choix politique qui s'appuie sur la reconnaissance des différences ou encore sur ce qui est aujourd'hui appelé des politiques d'identité (identity politics). Cela consiste à promouvoir les spécificités culturelles et à assurer leur représentativité égale dans la communauté politique. Par conséquent, le multiculturalisme se réfère avant tout à l'État-nation qui, dans ses principes, tend vers l'unification territoriale, linguistique et culturelle. Il a été considéré comme une réponse - pragmatique ou idéologique à la gestion de la diversité propre à toute société industrielle et aux moyens de l'inclure dans la communauté politique.

Défendant le principe de la reconnaissance des particularités culturelles par des institutions publiques, il constitue, depuis les années 80 , l'un des thèmes les plus débattus dans le domaine des sciences sociales, toutes disciplines confondues ${ }^{2}$. Pour certains, le multiculturalisme correspond au respect des identités culturelles, à l'égalité des droits et des chances, et constitue le fondement de la démocratie; pour d'autres, au contraire, il s'apparente au «tribalisme » et, de ce fait, remet en cause l'intégrité et l'unité nationale assurées, jusque-là, par l'État. Pour certains, il sert à contrecarrer le nationalisme et, pour d'autres, à l'inverse, il est à l'origine des sentiments et des expressions nationalistes.

Les débats opposent surtout ceux qui défendent une vision libérale - respect de la liberté individuelle face à une approche communautaire - et républicaine de la société «pluraliste » sur le terrain de la justice sociale ${ }^{3}$. Dans l'ensemble, l'analyse du multiculturalisme pose la question fondamentale quant à l'idéologie universelle représentée par l'État-nation qui s'oppose au particulier, d'une part, et à la défini-

2. Voir le débat autour de Charles Taylor dans les textes réunis par Amy Gutman, The Politics of Recognition. Princeton University Press, Princeton, NJ, 1992 (1 1 tre édition).

3. Voir aussi Jan Raz, "Multiculturalism: a Liberal Perspective ". Dissent, hiver 1994, p. 67-79. 
tion d'un espace civique commun de participation politique, de l'autre. Cela transforme une simple analyse anthropologique de la diversité culturelle en vision idéologique du pluralisme où la société est présentée comme un terrain d'affrontement des valeurs culturelles qui se transforment en intérêts particuliers dans le domaine politique, et non plus celui de la recherche de l'intérêt général, faisant de la vie politique un espace où rivalisent des identités perçues comme majoritaires ou minoritaires ${ }^{4}$, en quête de représentativité.

Contrairement aux arguments qui avancent l'idée que le multiculturalisme concerne les relations entre les groupes dits ethniques, l'allure passionnée du débat témoigne surtout de son association à une analyse sur l'unité nationale et à la définition de son identité. En réalité, les débats mettent en évidence le lien (ou l'absence de lien) entre la réalité sociale et l'idéologie de la construction de l'État-nation, conduisant ce dernier à redéfinir ses principes d'inclusion, d'universalité et de citoyenneté, bref à se redéfinir. Plus encore, le multiculturalisme comme concept et comme politique appliquée se trouve au cœur des interactions entre populations immigrées ou minoritaires et les États, conduisant ces derniers à des négociations identitaires de part et d'autre ${ }^{5}$. Dans cette perspective, le multiculturalisme pourrait surtout constituer une théorie permettant aux États-nations de trouver un nouvel équilibre entre les structures communautaires émergentes et les institutions nationales. La même question se pose dans le cadre de la construction européenne : le multiculturalisme, un fait dû au départ à la diversité identitaire des États-nations qui la constituent, pourrait être analysé comme une théorie fondatrice d'une identité européenne ${ }^{6}$.

\section{Le mot et le débat}

Le terme de multiculturalisme nécessite une contextualisation de son usage comme de sa pratique ${ }^{7}$. Le concept, né au Canada,

\footnotetext{
4. Le concept de minorité doit être pris avec beaucoup de précaution. Tout d'abord, la minorité dans le contexte européen vaut une nation et se réfère à un peuple. Voir, à ce sujet, l'analyse historique de la naissance des minorités : Guy Hermet, Histoire des nations et du nationalisme en Europe, Le Seuil. Paris, 1996. 5. Riva Kastoryano, La France, l'Allemagne et leurs immigrés. Négocier l'identité. Armand Colin. Paris, 1996. 6. Riva Kastoryano. Quelle identité pour l'Europe ? Le multiculturalisme à l'épreuve, Presses de Sciences Po, Paris, 1998.

7. Voir Jan Raz, article cité (3), Jean Leca, "La démocratie à l'épreuve du pluralisme ", Revue française de science politique, vol. 46, $n=2,1996$, p. 225-279.
} 
s'accompagne d'une politique appelée par Charles Taylor la «politique de la reconnaissance " qu'il définit comme "la défense démocratique de la diversité culturelle dans une perspective universaliste ${ }^{8}$. Il trouve une légitimité politique dans le multiculturalisme constitutionnel au Canada porté par la Charte des droits et libertés et admis officiellement comme la caractéristique fondamentale de l'État canadien'.

Le terme fait son chemin en Amérique du Nord depuis les années 60 en réponse à une "demande de reconnaissance " des minorités ethniques. Il trouve son fondement dans le mouvement des droits civils et prend forme avec la mise en place, dès 1965, des mesures d'« affirmative action ». Traduit en français comme « discrimination positive » à partir de l'interprétation du sociologue Nathan Glazer, ces mesures consistent à réduire les inégalités raciales ou autres en cherchant à réparer les méfaits des politiques passées, notamment de l'esclavage et de la ségrégation raciale. Ce qui, jusque-là, faisait partie du «pluralisme culturel " défini par Milton Gordon comme une théorie explicite de la nation américaine ${ }^{10}$, se transforme, dans les années 80 , en multiculturalisme accompagné de programme d'enseignement dispensé et de langage politically correct, et devient source de polémiques dans les universités, dans la vic politique et dans les médias opposant les défenseurs d'une "politique des différences" à ceux qui se soucient avant tout du lien social. Il s'agit de part et d'autre de s'interroger sur les conséquences du multiculturalisme sur l'unité nationale de l'Amérique. Pour les uns, il divise la société en micro-sociétés, la nation en nations; il est à l'origine des conflits ethniques, de la "désunion de l'Amérique", pour reprendre l'expression d'Arthur Schlesinger Jr. et, de ce fait, se trouve en contradiction avec le principe fondateur de la nation américaine exprimé par la formule E Pluribus Unum, "l'unité à partir de la diversité ». En revanche, d'autres voient dans le multiculturalisme et son application une ouverture de la société américaine vis-à-vis des exclus de l'assimilation, et perçoivent les effets pervers du multiculturalisme comme l'échec de l'assimilation, notamment des Noirs ${ }^{1}$.

8. Charles Taylor, Multiculturalism and the Politics of Recognition, Princeton University Press, Princeton, 1992

9. Philip Resnik. Thinking English Canada, Toronto, 1994 (voir plus spécifiquement le chapitre, 7).

10. Milton M. Gordon, Assimilation in American Life. The Role of Race, Religion and National Origins, Oxford University Press, New York, 1964.

11. Voir le dernier ouvrage de Nathan Glazer. We Are All Multiculturalists Now, Harvard University Press, Cambridge, MA, 1997 
La diversité des situations a conduit le philosophe canadien Will Kymicka à faire une distinction entre ce qu'il appelle les États multinationaux - constitués d'entités nationales linguistiquement et territorialement définies - et les États polyethniques, c'est-à-dire ceux où coexistent plusieurs communautés ethniques nées de l'immigration ${ }^{12}$. Bien que l'exemple du Canada corresponde à ces deux «types » à la fois et que le cas des Noirs aux États-Unis ne se réduise pas à l'immigration classique, cette distinction suggère une réflexion différenciée sur le multiculturalisme en fonction des structures de l'État.

En effet, en Europe, le multiculturalisme correspond à des situations diverses selon la formation de l'État et sa politique quant à la reconnaissance des particularités régionales et linguistiques. Certains pays du Vicux-Continent, comme l'Italic et l'Espagne, ont institutionnalisé le pluralisme en créant des régions dotées de pouvoirs, d'autres ont construit l'État sur un pluralisme linguistique, telles la Belgique et la Suisse ${ }^{13}$, avec, dans les deux cas, des communautés linguistiques et territoriales dotées de leurs propres institutions. Mais, dans des pays comme la France, l'Allemagne, la Grande-Bretagne, les Pays-Bas, le multiculturalisme renvoic, comme aux États-Unis, à la forme d'organisation supposée communautaire des populations issues de l'immigration des années 60, autour d'une nationalité ou d'une religion commune (ou les deux) et à la revendication de leurs spécificités dans la sphère publique.

Différents contextes, différentes références, différentes approches. $\mathrm{Si}$ au Canada, le multiculturalisme s'adresse aux " minorités " territoriales et linguistiques, aux États-Unis, il renvoie aux populations «exclues» de l'assimilation, en l'occurrence aux Noirs. Le souci d'égalité entre groupes a néanmoins étendu l'application du terme aux femmes, aux homosexuels, à toute collectivité qui remet en cause une démocratie égalitaire. En Europe occidentale, l'usage du terme " multiculturalisme " marque le passage d'une immigration économique temporaire à une présence permanente des populations immigrées des années 60 , notamment de celles en provenance des pays musulmans,

12. Will Kymicka, Multicultural Citizenship, Oxford University Press, New York, 1995 (voir plus spécialement le chapitre 2. "Politics of Multiculturalism ").

13. Pour la Suisse, se référer a Uli Windisch, notamment "Les frontières linguistiques ", communication pré sentée au colloque "Vers un multiculturalisme européen ", CERI, FNSP, Paris, mars 1994. 
perçues comme les plus "étrangères " à une "culture " définie comme occidentale et surtout perçue comme une menace au sécularisme considéré comme valeur universelle contre les formes d'organisations communautaires particularistes fondées sur la religion et, de ce fait, pose la question de la reconnaissance et de la représentation institutionnelle.

Des similitudes dans les faits, jusqu'à un certain point, entraînent donc un recours aux mêmes concepts qui, utilisés dans des contextes nationaux différents, nécessitent une nouvelle définition; car les concepts voyagent mal. Ils sont chargés d'idéologie. Ils traînent avec eux le poids des traditions politiques et culturelles, l'histoirc de la formation des États et celle des populations qui les composent. Cela apparaît dans la rhétorique - ambiguë - sur le multiculturalisme qui renvoie parfois à un constat et à une prise de conscience de la diversité culturelle, religieuse, linguistique propre à toute société, mais qui constitue en même temps le fondement d'un discours défensif sur l'universalité de l'État-nation et de la communauté politique, seule porteuse de légitimité. C'est ainsi que le multiculturalisme est perçu comme un "spectre ${ }^{14}$ dans le débat intellectuel français, une idéologie qui renvoie à la "ghettoïsation" des minorités, situation attribuée au contexte américain considéré comme un antimodèle pour la France. En Allemagne, c'est "l'auto-cthnicisation des minorités» comme résultat du multiculturalisme qui est dénoncée ${ }^{15}$.

Malgré ces réactions, dès les années 80 , les médias comme la classe politique qualifient la société française de «multiraciale», «multiculturclle », "plurielle », " pluriculturelle ». En Allemagne, à la même période, le terme de multiculturalisme se répand de façon identique. Il se crée même à la municipalité de Francfort un secteur des «Affaires multiculturelles » dont le responsable, en même temps maire adjoint de la ville, Daniel Cohn-Bendit, prône une «démocratie multiculturelle" qui s'inspirerait du contrat social de Rousseau ${ }^{16}$. Mais aussi bien en France qu'en Allemagne, l'usage du terme a pour fonction un

14. Voir Esprit, juin 1995.

15. Frank-Olaf Radtke. "Multiculturalism in Germany ", communication présentée au colloque "Vers un multiculturalisme européen ". CERI, FNSP. Paris, mars 1994.

16. Ses ambitions et ses intentions sont développées dans un ouvrage qu'il a écrit en 1992. Voir Daniel Cohn-Bendit et Thomas Schmid. Heimat Babylon. Das Wagnis der Multikulturellen Demokratie. Hoffman and Campe. Hambourg, 1992. 
constat et une stratégic de représentation d'une société où coexistent des populations aux nationalités et aux religions différentes. En France, dans un souci de société démocratique où règnent les principes de l'égalité des droits, ces discours ont pour objectif de faire accepter par l'opinion publique la diversité (anthropologique) comme un fait inhérent à toute société moderne. En Allemagne aussi, cela apparaît, de la part des militants et des porte-parole des partis d'opposition, comme une façon de faire prendre conscience à l'opinion publique et à la classe politique en général que les "étrangers sont là pour rester ", que l'Allemagne est en fait un pays d'immigration, et de fait une société multiculturelle.

En Grande-Bretagne, le même constat avait déjà donné lieu à des élaborations théoriques autour de "race relations ${ }^{17}$ qui, dans les années 80 , constituent une base pour le multiculturalisme à la fois comme théoric et pratique. Son usage se confirme dans ce qui est appelé en France le modèle anglo-saxon, attribué surtout aux ÉtatsUnis en raison du statut accordé aux "minorités ethniques ", statut légitimé aussi en Grande-Bretagne et qui, surtout comme aux États-Unis, trace une ligne de démarcation en termes «raciaux». Aujourd'hui, la notion d'une "Grande-Bretagne multiculturclle » dénote, d'après Harry Goulbourne, "la reconnaissance de l'existence d'une citoyenneté composée de gens de couleur différents et célébrant la diversité culturelle " ${ }^{18}$. La mobilisation des populations musulmanes originaires de la péninsule indienne et leur demande de reconnaissance institutionnelle ont ajouté une dimension confessionnelle au multiculturalisme britannique ${ }^{19}$, tout comme en France ou en Allemagne où l'islam se trouve explicitement au cœur des débats sur la reconnaissance, comme d'ailleurs aux Pays-Bas et en Belgique.

Ainsi se rapprochent les pays européens dans leur discours sur le multiculturalisme, bien que tous ne lui accordent pas la même définition. Il s'agit, en France, d'intégrer les différences dans les structures institutionnelles de l'État avec un rappel banalisé des traditions politiques républicaines qui récusent toute représentation communautaire des

17. John Rex, Race Relations in Sociological Theory, Routledge, Londres, 1983.

18. Harry Goulbourne. Race Relations in Britain Since 1945. MacMillan, Londres, 1998, p. 25

19. Voir Tariq Modood, communication presentée à la conférence sur "Multiculturalism and Struggle for Recognition in Comparative Perspective ". Harvard University, mars 1999. 
populations dans un État qui ne reconnaît que l'individu comme interlocuteur. Pour la Grande-Bretagne, il pose la question de la représentativité et de l'égalité entre les «minorités raciales, ethniques et religieuses »; pour l'Allemagne, d'après Frank-Olaf Radtke, le discours sur le multiculturalisme apparaît comme une alternative à l'Ausländerpolitik, et marque ainsi un changement dans les codes de description de la société et la pratique institutionnelle vis-à-vis des populations immigrées ${ }^{20}$. Dans l'ensemble, l'appropriation d'un tel discours conduit à un dilemme entre intégration et reconnaissance, et des actions politiques qui lui donnent forme, qui l'adoptent ou la rejettent, mais surtout la justifient.

\section{Le multiculturalisme appliqué : controverses et paradoxes}

La recherche de l'égalité de traitement, suggérée par la politique de la reconnaissance, rapproche aussi les pays européens dans une sorte de multiculturalisme appliqué. Cela implique désormais l'extension de l'État-providence à un nouveau domaine - celui des identités -, avec la mise en place de politiques sociales en vue "d'assurer l'intégration » des populations issues de l'immigration ou des minorités (selon les cas) dans la société globale. Les États démocratiques sont ainsi conduits à définir des politiques qui tiendraient compte des différences culturelles, à travers les programmes scolaires (tel l'enseignement bilingue), la création d'associations ou de commissions municipales, par exemple.

Aux États-Unis, c'est dans le domaine de l'enseignement que le multiculturalisme est le plus controversé. Le principe du respect mutuel entre les groupes et les citoyens a imposé une réécriture de l'histoire avec une réinterprétation ethnique ou raciale de l'origine des civilisations ${ }^{21}$. En Europe, cette exaltation des origines s'exprime à travers les associations. En France, "le droit à la différence ", accompagné dès 1981 de la libéralisation de la loi sur les associations d'étrangers, a

20. Frank-Olaf Radtke, article cité (15).

21. L'exemple le plus connu est fourni par l'ouvrage The Origins of African Civilization qui prétend que l'ancienne Egypte appartient a la civilisation noire. que toutes les races descendent de la race noire, et qu'il en est de même pour la civilisation occidentale puisqu'elle dérive de la civilisation egyptienne. Des spéculations similaires alimentent un autre ouvrage. publié en 1987. Black Athena: The Afroasiatic Roots of Classical Civilization. Le titre est assez explicite de la volonté avec laquelle les Noirs américains entendent exprimer leur fierté ethnique et raciale. 
donné un statut légal aux organisations privilégiant les identités, qu'elles se définissent comme principalement sociales, culturelles, laïques ou religieuses. En Grande-Bretagne, la Commission pour l'égalité des races (Commission for Racial Equality) a promulgué, en 1976, le Race Relations Act. L'objectif principal était de lutter contre le racisme, d'éliminer les discriminations, d'assurer une égalité des chances et d'établir ainsi de bonnes relations entre différents " groupes raciaux ${ }^{22}$. La société britannique est désormais définie comme une société multiculturelle et s'accompagne de programmes d'enseignement (le rapport Swann: Education for All) dont le but est de souligner les valeurs communes et d'assurer la cohésion sociale ${ }^{23}$. Aux Pays-Bas, toujours dans les années 80 , se définit une politique de minorités qui a pour objectifs de "promonvoir le multiculturalisme et l'émancipation des communautés ethniques » ainsi que leur représentation politique ${ }^{24}$.

L'application de telles mesures a été à l'origine d'une cascade d'effets pervers qui mettent en évidence une série de paradoxes des politiques privilégiant la culture et les identités, et valorisant les différences dans la recherche de l'égalité. Ces paradoxes relèvent en fin de compte de ceux de la démocratic. Comment choisir ou articuler entre le droit individuel et collectif ? Comment avancer une idéologie universaliste fondée sur l'individu et les droits de l'homme, et appliquer un traitement différencié aux groupes ? Comment combiner différence et égalité ${ }^{25}$ ?

Toutes ces questions et approches mettent l'accent sur les conséquences controversées d'une politique de la reconnaissance: la fragmentation de la société en communautés repliées sur elles-mêmes, une identification différenciée à la communauté politique qui défic le sens civique de la nation, et enfin une politique des différences qui, appliquée par les États, conduit à un clientélisme des actcurs politiques et remet en cause le libre choix des individus ${ }^{26}$. Plus encore, le

22. John Crowley, Immigration, "relations raciales " et mobilisations minoritaires au Royaume-Uni. La démocratie face à la complexité sociale, thèse d'ètudes politiques, sous la direction de Jean Leca, Paris, 1994.

23. Harry Goubourne, "British Multi-culturalism and the Communal Option ", communication présentée au colloque "Vers un Multiculturalisme européen ". CERI, FNSP, Paris, mars 1994.

24. Han Entzinger, " $Y$ a-t-il un avenir pour le modèle néerlandais des "minorités ethniques "? ", Revue européenne des migrations internationales, Vol. 10, $\mathrm{n}$ \% 1, 1994, p. 73-95.

25. Voir à ce propos Joan W. Scott, "The Conundrum of Equality ", Occasional papers from the School of Social Science, Institute for Advanced Studies, Princeton, mars 1999, paper $n^{\prime} 2$

26. Alexandra Alund et Karl Schierup. Paradoxes of Multiculturalism. Avebury. Aldershot, 1991. 
multiculturalisme comme choix politique contribue, dans une large mesure, à définir ou à cibler « la population » perçue la plus étrangère à la représentation identitaire de la communauté nationale, accentuant ainsi les particularismes de part et d'autre ${ }^{27}$.

Mais la réflexion sur le multiculturalisme, loin d'apporter des réponses à ces questions émanant de la réalité sociale, se concentre surtout sur une approche normative de philosophie et d'éthique politique opposant libéraux et communautariens sur la définition et l'application de justice sociale et de citoyenneté, d'inclusion et de participation. Contrairement à l'égalité entre individus-citoyens et leur participation civique à la communauté nationale au-delà des considérations identitaires, le multiculturalisme associé au communautarisme considère la reconnaissance des différences identitaires comme caractéristique d'une société juste, et la représentation comme un moyen d'inclusion et de participation d'un groupe dans la communauté nationale, à travers une appartenance culturelle organisée autour des associations ou autres.

En France, par exemple, dans les années 80, les dispositifs en faveur d'une intégration sociale des populations immigrées ont reposé en grande partie sur une aide publique destinée à permettre à la diversité culturelle de s'organiser et aux identités de s'affirmer. En République fédérale, où l'étranger est perçu avant tout dans son appartenance à un groupe ethno-national, les programmes d'intégration visent à les inclure dans le corporatisme allemand en tant que vecteur de communautés d'intérêts. Ainsi, dans les deux pays, les politiques destinées à faire disparaître les inégalités sociales issues de différences culturelles ont fait apparaître les identités comme scules bases légitimes d'action politique. Dans cette perspective, la formation préalable en communautés, cette fois ethno-culturelle et religicuse, est devenue une tactique nécessaire pour aboutir à une reconnaissance légale ultérieure des particularités ainsi affichées et pour négocier celles-ci avec le pouvoir. La politisation des identités exprimées dans le cadre des associations a done conduit une partie des populations issues de l'immigration à développer des instruments de participation politique et à revendiquer la reconnaissance «d'une communauté » qui se construit dans la relation à l'État. 
Pris dans un processus d'interaction permanente, les États ont été amenés dès lors à rappeler, de leur côté, les valeurs fondatrices de la nation dont ils sont toujours les gardiens, et à les négocier avec des populations qui exprimaient d'autres identifications et allégeances. Mais, en retour, cela a conduit ces derniers à définir plus fermement les éléments identitaires à partir desquels elles pouvaient se constituer en communauté dans leur quête de reconnaissance dont les limites s'inscrivaient dans le cadre de la légitimité des États de résidence; ce qui amène les Maghrébins à définir des solidarités autour de l'islam face à la laïcité en France, et les ressortissants turcs à se constituer en minorité ethnique face à la conception de nationalité allemande.

La réalité sociale émane donc des interactions entre États et populations concernées par les politiques d'identité. Les sociétés démocratiques, qui ont développé un discours sur les différences et l'égalité, et pratiqué des mesures «multiculturelles», se trouvent face à des formes de participation de plus en plus collective et d'engagement des individus tant à l'intérieur d'une communauté culturelle, ethnique ou religieuse ou une "communauté de circonstance», pour reprendre l'expression de Jean $\mathrm{Leca}^{28}$, qu'à l'intéricur de la communauté nationale. La question se pose désormais de savoir comment assurer une participation des «exclus de l'assimilation » par le biais d'un groupe reconnu comme « légitime » dans le cadre institutionnel de l'État; et comment établir un nouvel équilibre entre les structures dites communautaires et les institutions nationales.

Ainsi le multiculturalisme, au-delà d'une dispute entre une vision libérale ou communautaire de participation, pose la question d'une représentation institutionnelle dans le cadre de la légitimité de l'État. Une telle approche, qui met en évidence une «assimilation institutionnelle », renvoie à une identification politique différenciée, à la fois à une communauté culturelle et à la communauté nationale, sans que cela soit en contradiction. L'idée principale est de définir un espace civique de participation politique.

\section{Vers un multiculturalisme européen ?}

La définition d'un espace commun de participation politique se trouve précisément au cœur de la construction d'une Europe politique. Malgré des situations parallèles et les convergences politiques entre les 
pays membres de l'Union, apparaît une différenciation de plus en plus prononcée du discours étayé par le sentiment national, exprimé par des «modèles nationaux », notamment dans l'équation entre peuple, nation et État, ou encore entre minorité et majorité qui n'ont bien entendu pas le même sens dans différents pays européens. L'élaboration de ces références nationales révèle des paradoxes et des ambiguïtés dans les projets politiques de la construction européenne. Elle souligne d'abord la prédominance des particularités nationales, mais aussi la nécessité de les redéfinir dans un espace politique supranational qui accroît les interactions.

De toute évidence, l'Europe unic relève, dès ses origines, d'un pluralisme de fait : diversité linguistique et culturelle - nationales et régionales, majoritaires et minoritaires -, diversité institutionnelle, où chacune porte la charge de fortes traditions culturelles et politiques. Le projet politique européen ne peut ignorer cette pluralité au sein de laquelle s'expriment et s'imposent les différentes cultures nationales.

Mais s'interroger sur un nouvel espace politique revient à s'interroger sur la constitution d'un nouveau modèle de société. Un modèle pluraliste naturellement fondé sur des principes redéfinis par l'apport de différentes cultures nationales et/ou minoritaires, qui se revendiquent comme nation, pour former une culture commune européenne. Les interrogations portent sur le mode de participation et de représentativité des individus ou des groupes et sur les moyens d'expression de toutes les identités collectives, aussi complexes et hétérogènes soientelles. À cela s'ajoutent les étrangers «non européens » résidant en Europe. Même si les politiques d'immigration et d'intégration relèvent des compétences nationales, les populations issues de l'immigration, qui affichent d'autres appartenances et d'autres espaces de participation que ceux des États-nations de résidence, trouvent un appui dans ce nouvel espace politique en construction, à l'identité incertaine, pour promouvoir des identités collectives dites «d'origine", qu'elles soient religieuses ou nationales. L'affaiblissement des identités nationales, voire leur repli, combiné à la mise en ouvre des projets politiques communs, entraîne une mobilisation pour la représentation des identités «minoritaires» dans l'espace européen qui se cherche de nouvelles références. 
La question qui se pose est de savoir s'il est vrai que le "multiculturalisme » est à la base de divisions au sein des États-nations; ne pourrait-il pas être aussi à l'origine d'une identité européenne : identité produite par des instances juridiques qui mettraient en évidence les échanges culturels et politiques; identité organisée autour des réseaux de communication formels et informels entre différents groupes nationaux et non nationaux ? Cela pose d'emblée la question de la citoyenneté européenne, celle de l'intervention des institutions supranationales dans la définition et l'élaboration du concept de citoyenneté et d'identité en Europe pour aboutir, au-delà de sa définition juridique, à la formation d'une culture politique européenne ${ }^{29}$.

Se poser la question du multiculturalisme dans le cadre de l'Union revient justement à s'interroger sur l'émergence d'une communauté politique européenne, avec une culture politique commune à toutes les nations qui forment cette nouvelle entité et à tous les États-membres qui s'imposent avec leur histoire, leurs traditions et leurs valeurs, bref sur la définition d'une identité publique, pour reprendre l'expression de John Rawls. La tâche principale de l'Europe pourrait dès lors être définic comme la gestion de la diversité des cultures politiques dans le cadre d'une démocratic universelle par la mise en place d'un espace public unifié, un espace de production de pouvoir politique curopéen et un espace de citoyenneté, mais en même temps un espace de participation et d'identification commune, bref un espace de citoyenneté, une citoyenneté qui serait le moteur d'une identité curopéenne et un espace de représentation de l'intérêt collectif.

De nombreux débats sur la citoyenneté, la nationalité et l'identité européenne ont accompagné la transformation progressive d'un grand marché unique en un espace politique et ont souligné la multiplicité des références identitaires dans la formation d'une Europe politique. Jean-Marc Ferry propose un modèle «post-national» pour marquer le dépassement du "principe nationaliste » entraîné par la construction d'une Europe politique ${ }^{30}$. Jürgen Habermas, de son côté, développe le concept de «patriotisme constitutionnel » pour souligner la séparation entre le sentiment d'appartenance qu'implique la citoyen-

29. Riva Kastoryano, op. cit. (6).

30. Jean-Marc Ferry, "La pertinence du post-national " dans Jacques Lenoble et Nicole Dewandre (dir.), L'Europe au soir du siècle : identité et démocratie, Esprit, Paris, 1992, p. 39-59. 
neté nationale et sa pratique juridique au-delà des cadres de l'Étatnation. Pour lui, la citoyenneté «est conçue sur le modèle d'affiliation à une organisation qui assure une position juridique et situe l'individu en dehors de l'État".

De même, les registres d'appartenance et d'engagement politique montrent que la pratique de la citoyenneté fait apparaître une multiplicité d'intérêts ainsi que d'appartenances et d'allégeances dans le cadre européen et se détache d'une conception exclusivement liée à l'identité nationale. Mais, en même temps, l'Europe, en tant qu'espace ouvert à la revendication des intérêts et des identités de ses citoyens comme de ses résidents, a stimulé un mode de participation transnationale qui leur permet d'affirmer une autonomie par rapport aux systèmes étatiques territorialement définis. C'est dans cette logique que les populations issues de l'immigration, résidant dans un pays européen, entreprennent une action transnationale pour renforcer leur revendication d'égalité des droits et de traitement au niveau européen ainsi que leur lutte contre le racisme. Un tel engagement témoigne d'une volonté - ex post - de participation à la formation d'une identité européenne qui se situerait au-delà des identités nationales.

C'est là un des paradoxes de la construction européenne ${ }^{31}$. En effet, les réseaux transnationaux constituent un moyen de contourner les politiques nationales. Ils contribuent, à certains égards, à la formation de «communautés transnationales» qui dépassent le cadre d'États-nations. Mais, en réalité, ils apparaissent désormais comme des structures indispensables pour négocier avec les pouvoirs publics leur reconnaissance dans des cadres qui restent nationaux. La consolidation des solidarités transnationales vise en somme à influencer les États de l'extérieur. Ce constat est encore plus flagrant dans le cas des populations immigrées : la structuration transfrontalière des réseaux associatifs a pour objectif de renforcer leur représentativité au niveau européen, mais son but pratique est d'aboutir à une égalité de droits et à l'élimination de toute discrimination au niveau national.

31. Riva Kastoryano, "Participation transnationale et la citoyenneté. Les immigrés dans l'Union européenne ". Culture et conflits, n" 28, hiver 1997, p. 59-75; voir aussi Christian Lequesne, "La Commission européenne entre autonomie et dépendance ", Revue française de science politique, vol. 46. n" 3. juin 1996. p. $389-408$. 
Ainsi, un espace public européen semble se dessiner en dehors des États-nations comme le démontrent la structuration des réseaux et l'engagement politique des acteurs, bien que les intérêts soient, a priori, particuliers. Mais c'est surtout dans l'interpénétration entre les États et l'Union européenne que se jouent le pouvoir politique et les influences réciproques, et dans les nations que s'exprime l'intérêt général faute d'espace politique européen commun. Ce qui conduit à considérer les États comme la force structurante de la construction curopéenne.

Or, l'une des réflexions sur l'Europe unic porte justement sur le dépassement des modèles étatiques perçus comme particularistes, et sur les moyens de relier les différents espaces juridiques, culturels et politiques qui la composent. Cela suppose une production de normes culturelles et juridiques où les intérêts des États scraient exprimés, leurs principes et leur souveraineté protégés, leur identité représentéc, bref, un modèle de société pluraliste avec une Constitution fondée sur des principes restructurés par la reconnaissance des différentes cultures pour former une culture politique commune curopéenne. Il faut alors s'interroger sur les façons de combiner la pluralité des cultures nationales et l'unité politique nécessaire pour définir une identité curopéenne. De nouvelles formes de démocratic sont à imaginer : Chantal Mouffe propose une « démocratie plurielle» qui tiendrait compte de cette vision multiculturelle des réalités politiques, cherchant à trouver une nouvelle forme d'articulation entre l'universel et le particulier ${ }^{32}$.

Mais la dynamique de formation d'une culture politique partagée au scin de l'Union ne peut se déployer que dans le mouvement de confrontation entre des traditions nationales différentes. Déjà dans le cadre national, les relations accrues entre les populations immigrées, structurées de plus en plus en communautés porteuses d'identités spécifiques, témoignant cependant d'une «acculturation politique » - pour reprendre l'expression utilisée par Habermas - dans leur forme de participation et d'adhésion à la culture civique ambiante, conduisent les États à des négociations d'identité qui remettent en cause les

32. Chantal Mouffe, "La démocratie entre modernité et post-modernité : pour une démocratie plurielle ", Revue du MAUSS, $n * 8$, deuxième trimestre 1990, p. 14-30. 
traditions politiques des unes et des autres dans l'espoir d'aboutir à un nouveau compromis historique. De même, dans le cadre européen, le «multiculturalisme» ne peut-il être à l'origine d'une nouvelle articulation aboutissant à un compromis ? Le multiculturalisme, comme fondement d'une unité politique tenant compte de la diversité culturelle, politique et juridique qui caractérise l'Europe, pourrait-il permettre de dépasser les tensions et les rapports de force entre les États-membres d'un côté, entre États-membres et Bruxelles de l'autre, comme le suggère la réflexion sur une Europe confédérale, et d'aboutir à une unité politique dans le respect de la multiplicité constitutionnelle, ainsi que dans la diversité identitaire de l'Europe ${ }^{33}$ ? En même temps, contrairement au fédéralisme qui s'appuie sur l'unité territoriale et politique et sur la volonté du peuple au départ, pour aboutir à la définition d'une Constitution commune, l'idée de multiculturalisme emprunte le chemin inverse, à savoir, partir du multiple pour arriver à une unité politique en cherchant ainsi à rétablir un nouvel équilibre entre culture, politique et territoire, et à susciter à la longue une identification, de la part des acteurs, avec une nouvelle entité politique qui tiendrait compte des traditions politiques diverses.

De toute évidence, l'Europe comme espace de citoyenneté, d'engagement et de participation, comme espace d'appartenance régionale et nationale à la fois, voire ethnique et religieuse, ajoute un nouvel élément au choix identitaire de l'individu: la référence européenne. Le multiculturalisme, comme base de la négociation de multiples identités, ne pourrait-il répondre aux problèmes de l'allégeance en permettant de penser l'Union européenne non à partir d'une construction sur le modèle d'État-nation, mais d'une réflexion sur la coexistence des identités qui la composent? 